
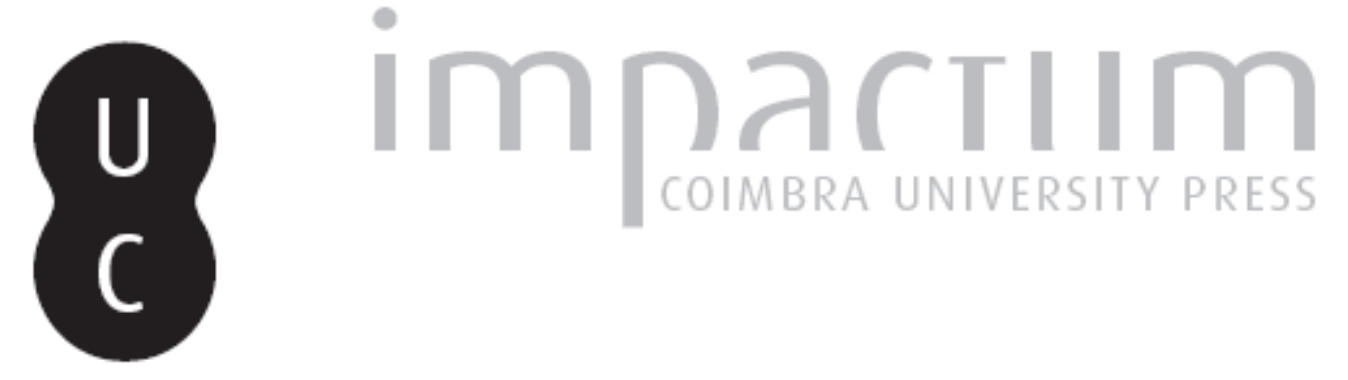

\title{
Wenceslau de Moraes e la Grande Guerra vista dal Giappone
}

Autor(es): $\quad$ Losano, Mario G.

Publicado por: Imprensa da Universidade de Coimbra

URL persistente: URI:http://hdl.handle.net/10316.2/42415

DOI: $\quad$ DOI:https://doi.org/10.14195/0870-8584_9_9

Accessed : $\quad$ 26-Apr-2023 14:05:07

A navegação consulta e descarregamento dos títulos inseridos nas Bibliotecas Digitais UC Digitalis, UC Pombalina e UC Impactum, pressupõem a aceitação plena e sem reservas dos Termos e Condições de Uso destas Bibliotecas Digitais, disponíveis em https://digitalis.uc.pt/pt-pt/termos.

Conforme exposto nos referidos Termos e Condições de Uso, o descarregamento de títulos de acesso restrito requer uma licença válida de autorização devendo o utilizador aceder ao(s) documento(s) a partir de um endereço de IP da instituição detentora da supramencionada licença.

Ao utilizador é apenas permitido o descarregamento para uso pessoal, pelo que o emprego do(s) título(s) descarregado(s) para outro fim, designadamente comercial, carece de autorização do respetivo autor ou editor da obra.

Na medida em que todas as obras da UC Digitalis se encontram protegidas pelo Código do Direito de Autor e Direitos Conexos e demais legislação aplicável, toda a cópia, parcial ou total, deste documento, nos casos em que é legalmente admitida, deverá conter ou fazer-se acompanhar por este aviso.

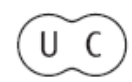




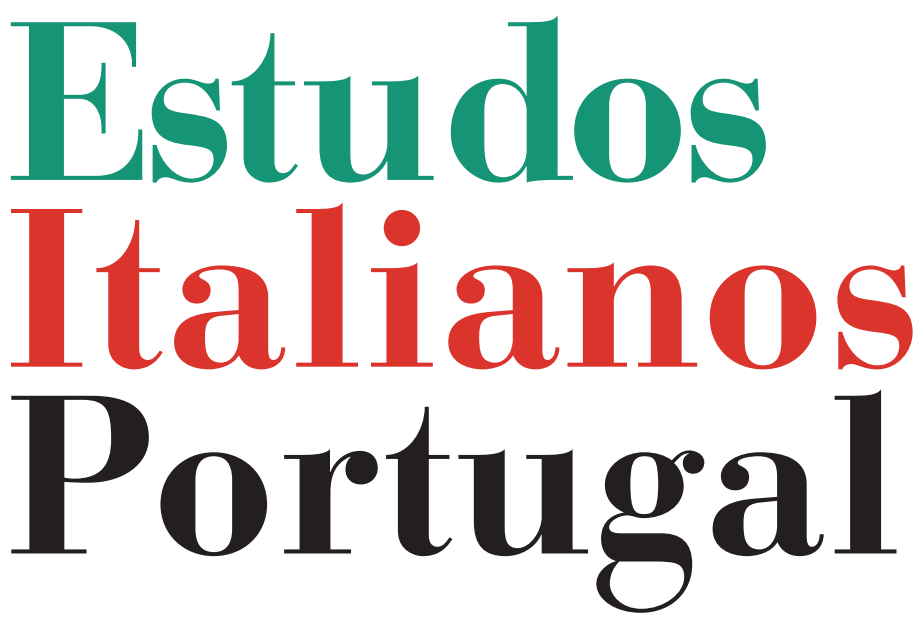

Instituto

Italiano

de Cultura

de Lisboa

Nova Série

$\mathbf{N}^{\circ} 9$ 


\section{WENCESLAU DE MORAES E LA GRANDE GUERRA VISTA DAL GIAPPONE}

Mario G. Losano*

1. Wenceslau de moraes, una passione estrema per L'ORIENTE ESTREMO

Quando si parla di Wenceslau de Moraes (1854-1929) fuori dal Portogallo, è inevitabile il richiamo a Pierre Loti (1850-1923), ma in questo paragone Pierre Loti è il genus (cioè l'esotismo), mentre Wenceslau de Moraes è la differenza specifica. I due scrittori hanno in comune alcuni tratti esteriori: sono coetanei e le loro vite corrono parallele; entrambi sono ufficiali della marina militare e possono così scoprire il Giappone in un'epoca in cui quelle isole non erano raggiunte da alcun collegamento marittimo di linea; infine, la scoperta della civiltà giapponese li colpisce profondamente e ispira le loro opere più fortunate.

Qui finiscono però i pur rilevanti parallelismi. Per Loti il contatto con il Giappone fu momentaneo ed epidermico, ed egli guardò con superiorità al mondo Meiji sentendosi sempre e soltanto francese: dalle sue escursioni sulla terraferma nipponica ritornava a bordo cercandovi "un piccolo angolo

* Mario G. Losano ha insegnato filosofia del diritto e informatica giuridica nell'Università Statale di Milano e in quella del Piemonte orientale. A Torino insegna nella Scuola di Dottorato di quell'università ed è socio dell'Accademia delle Scienze. 
di Francia”. Moraes invece si trasferì in Giappone, vi si sposò e lì visse per oltre trent'anni senza più tornare in Portogallo, cercando un'identificazione con l'anima giapponese e nutrendo però fino all'ultimo un appassionato ricordo della terra natìa. Loti era sicuro di sé e del suo successo; Moraes era modesto fin quasi all'autolesionismo.

Queste differenze biografiche si riflettono anche nelle loro opere: il Giappone offre a Loti uno dei tanti scenari esotici per i suoi romanzi, dall'Islanda ai Tropici, dalla Turchia al Senegal; invece il tema pressoché costante degli scritti di Moraes è il riflesso del Giappone nella sua anima tormenta$\mathrm{ta}^{1}$.

2. PIÙ Di TRENT'ANNI DI LETTERE TRA GIAPPONE E PORTOGALLO

Nelle sue lettere Wenceslau de Moraes presuppone la conoscenza di eventi portoghesi e giapponesi sulla Grande Guerra che qui si possono soltanto accennare in estrema sintesi.

In Portogallo i preparativi bellici iniziarono già il 12 agosto 1914 , con l'invio di due distaccamenti in Angola e Mozambico perché i tedeschi minacciavano le colonie in Africa ${ }^{2}$. Tuttavia, su pressione degli inglesi, il Portogallo si dichiarò dapprima né belligerante né neutrale ed entrò in guerra soltanto nel 1916, quando requisì le navi tedesche che si trovavano in acque territoriali portoghesi. Da quel momento il "Corpo Expedicionário Português" operò nelle Fiandre a fianco degli inglesi, subendo un duro rovescio nella battaglia

${ }^{1}$ Mario G. Losano, "L'esotismo del portoghese Wenceslau de Moraes (1854-1929) tra Giappone eroico e Giappone industriale”, Rivista degli Studi Orientali, 86, 2013, pp. 121-151, e la letteratura ivi citata.

${ }^{2}$ Generale José Estevão Moraes Sarmento, $A$ expansão alemã. Causa determinante da guerra de 1915-1918. Suas tentativas e perigos na África Portuguesa, Lisboa, Guimarães, 1919. 
di La Lys, nella zona di Ypres. Parallelamente, circa 30000 soldati portoghesi operavano nelle colonie africane ${ }^{3}$.

Sul piano interno la situazione portoghese era agitata. Il 20 gennaio 1915 il "Movimento das Espadas" aveva portato al potere Pimenta de Castro, la cui dittatura durò solo cinque mesí. Il 5 dicembre 1915 gli succedette Sidonio Pais, che sospese i diritti costituzionali e morì vittima di un attentato nel 5 dicembre $1918^{5}$. Questa instabilità politica, l'aumento dei prezzi e il razionamento dei beni di consumo generarono un diffuso malcontento popolare che culminò nella "Revolução da Batata": dal 19 al 21 maggio 1917 a Lisbona e Oporto vennero assaltati i negozi e fu proclamato lo stato d'assedio.

Il Giappone entrò in guerra il 23 agosto 1914 contro la Germania e due giorni dopo contro l'Austria-Ungheria, con il compito di affrontare la marina tedesca e austriaca nel Pacifico e nell'Oceano Indiano. Poiché gli Alleati erano quasi totalmente occupati in Europa e col trasporto delle truppe degli Stati Uniti (entrati in guerra il 6 aprile 1917), i giapponesi godettero di una grande libertà d'azione su un vasto scacchiere. La marina giapponese occupò e gestì autonomamente fino al 1921 i possedimenti tedeschi in Micronesia (isole Marianne, Caroline e Marshall). Le mire del Giappone si estendevano anche alla Cina, dove occupò i possedimenti tedeschi. Già nella Grande Guerra, quindi, andava profilandosi quell'espansionismo giapponese che con la Seconda

\footnotetext{
${ }^{3}$ Ricavo questa e altre date dalla mostra Portugal e a Grande Guerra presso la Biblioteca Nacional de Lisboa (19 maggio - 31 luglio 2014).

${ }^{4}$ José Joaquim Pereira Pimenta de Castro (1846-1918) fu Ministro repubblicano della guerra per soli due mesi nel 1911 e poi Primo Ministro per cinque mesi, caratterizzati da una svolta autocratica (28 gennaio - 14 maggio 1915). La sua affannosa vita politica riflette le difficoltà interne della Prima Repubblica portoghese, nata il 5 ottobre 1910.

${ }^{5}$ Sidónio Bernardino Cardoso da Silva Pais (1872-1918), repubblicano e contrario all'entrata in guerra, divise la sua vita tra la carriera militare e quella politica, oltre ad essere stato docente a Coimbra. Con lui iniziano le degenerazioni autocratiche della repubblica portoghese.
} 
guerra mondiale sarebbe culminato nella "Greater East Asia Co-Prosperity Sphere"6. Sul piano interno, le ristrettezze e gli aumenti di prezzi provocarono anche in Giappone tensioni e scioperi, di cui si trova traccia nella seconda lettera di Wenceslau de Moraes.

\section{IL DIPLOMATICO WENCESLAU DE MORAES E L'ITALIA.}

Nel corso della sua attività consolare, Wenceslau de Moraes ebbe rapporti ufficiali con l'Italia, perché egli, come diplomatico portoghese, ne rappresentava gli interessi nel Consolato di Kobe, mentre l'Ambasciata d'Italia a Tokyo rappresentava quelli del Portogallo in Giappone. Il tema del rapporto con l'Italia affiora anche incidentalmente: "Dunque il Ministro del Brasile, nostro Incaricato d'Affari, fa l'offeso con Lei! Divertente. Ai miei tempi, il Ministro dell'Italia, anch'egli nostro Incaricato d'Affari, non era tanto suscettibile: io non gli ho mai fatto la corte, né lui ha mai litigato con me. Cose del Brasile...."7.

Nelle due lettere tradotte nel prossimo paragrafo la presenza dell'Italia in Guerra ritorna due volte. Nel 1915, quando il Portogallo non era ancora in guerra, Moraes giudicò "una canagliata” la tardiva belligeranza dell'Italia. Nella lettera del 7 ottobre 1919 accenna a Gabriele d'Annunzio, di cui era ancora vivo il ricordo del volo su Vienna del 9 agosto 1918, ma soprattutto l'eco dell'annessione di Fiume al Regno d'Italia il 12 settembre 1919: "L'Italia, con il suo poeta alla testa", contribuiva "a complicare la situazione".

\footnotetext{
${ }^{6}$ Mario G. Losano, La geopolitica del Novecento. Dai Grandi Spazi delle dittature alla decolonizzazione, Milano, Bruno Mondadori, 2011, pp. 97-113.

7 Tokushima, 9 febbraio 1919, Wenceslau de Moraes, Cartas do Extremo Oriente, org., intr. e notas de Daniel Pires, Lisboa, Fundação Oriente, 1993, p. 123.
} 


\section{LA GRANDE GUERRA VISTA DALL“EREMITA” DI TOKUSHIMA.}

Nella sterminata distesa delle lettere scritte da Wenceslau de Moraes, la Grande Guerra spesso compare solo per accenni. A José Gonçalves Paúl, militare di carriera e marito della sorella minore, scrive che "la situazione politica del paese si presenta tanto spaventosa quanto la guerra, o forse anche di più" . Altre volte, sulle cartoline, Moraes si limita ad accenni: "La guerra è finita! Auguri! Ora vedremo che cosa ci porta la pace!"”. A Cerveira de Albuquerque trasmette la sua tetra visione del futuro: "Credo che la guerra abbia provocato un'enorme ondata di demenza che ha invaso il mondo intero, il che spiega la gran quantità di guai avvenuti nel nostro paese e in tutto il pianeta, in questi ultimi tempi”"10.

Le due lunghe lettere che seguono sono la prima traduzione in italiano di un testo di Moraes. In esse colpisce come l'“eremita", il "detenuto" di Tokushima (com'egli stesso si definiva) fosse al corrente degli eventi europei nonostante la distanza da cui li osservava e come, proprio per questa distanza, ne avesse una complessiva visione geopolitica. Esse non sono facilmente reperibili neppure in portoghese e la stessa vicenda della loro pubblicazione ne spiega la rarità.

Dal 17 novembre 1914 al 12 novembre 1927 Wenceslau de Moraes tenne una regolare corrispondenza con Polycarpo de Azevedo (1862-1929), alto ufficiale di marina di sentimenti monarchici che ebbe vari incarichi in Angola, Guinea e Cina. In quest'ultima sede, Azevedo strinse amicizia con

\footnotetext{
${ }^{8}$ Tokushima, 26 marzo 1915, Wenceslau de Moraes, Cartas íntimas, pref. e anotações de Ângelo Pereira e Oldemiro César, Lisboa, Empresa Nacional de Publicidade, 1944, p. 99.

${ }^{9}$ Tokushima, 17 novembre 1918, Venceslau de Morais, Notícias do exílio nipónico, pref., transcrição, comentários e notas de Jorge Dias, Macau, Instituto Cultural de Macau, 1993, t. II, p. 977 (cartolina alla sorella minore Francisca Paúl).

${ }_{10}$ Tokushima, 6 giugno 1916, Wenceslau de Moraes, Cartas do Extremo Oriente, nota 7 , p. 96.

${ }^{11}$ Così Henriques Oliveira, "Explicação deste livro", p. 8 n.n. (cfr. nota 14).
} 
Pierre Loti e con Wenceslau de Moraes ${ }^{11}$. Le 23 lettere conservate dalla famiglia vennero offerte per la vendita a un antiquario di Lisbona, Arnaldo Henriques Oliveira, che verso il 1960 le incluse nel catalogo d'una sua asta. Le acquistò un bibliofilo lisboense, l'architetto Francisco dos Santos, "proprietario d'una opulenta e vasta biblioteca in cui figurano tutti i libri, gli scritti volanti e le curiosità"12 di Wenceslau de Moraes. Dato l'interesse di quelle lettere, l'antiquario e il bibliofilo decisero di pubblicarle.

La prefazione venne affidata ad Antero Carreiro de Freitas che, come Incaricato d'Affari del Portogallo in Giappone, aveva conosciuto Wenceslau de Moraes, lo aveva visitato a Tokushima, aveva assistito ai suoi funerali e si era infine occupato del suo testamento ${ }^{13}$. Però Carreiro de Freitas morì improvvisamente senza aver ultimato quella prefazione e l'addolorato "povero bouquiniste estensore di cataloghi" non si sentì di sostituirlo. "Questo libro non avrà, dunque, una prefazione", sono le parole con cui Henriques Oliveira conclude le sue brevi note introduttive al volume, i cui 500 esemplari videro la luce nel 1961 e sono oggi pressoché introvabili ${ }^{14}$.

Tokushima, 8 agosto $1915^{15}$.

Mio caro Amico, Signor Polycarpo de Azevedo,

Ricevo in questo momento la sua bella lettera del 23 giugno, con la quasi-promessa che fra breve riceverò altre sue

12 Così Henriques Oliveira, ib., p. 4 n.n. (cfr. nota 14).

${ }^{13}$ Antero Carreiro de Freitas (nato nel 1896) fu Ministro di 1.a classe e Capo del Protocollo del Ministero degli Affari Esteri portoghese. Morì tra la fine del $1960 \mathrm{e}$ l'inizio del gennaio 1961, secondo Henriques Oliveira (ib., p. 5 n.n.: cfr. nota 14).

${ }^{14}$ Wenceslau de Moraes, Cartas. dirigidas de Tokushima entre 1914 e 1927 ao seu grande amigo Polycarpo de Azevedo, ed. Arnaldo Henriques Oliveira Livreiro Antiquário, Lisboa 1961. Le due frasi citate sono alle pp. 6 e 5 n.n.

${ }^{15}$ La lettera qui tradotta è in Moraes, Cartas a Polycarpo de Azevedo (cfr. nota 14), pp. 15-18. 
notizie. (Tenga presente che c'è già la via Siberia, indicazione che è meglio mettere nell'indirizzo.)

Sì, signore, qui ho goduto della primavera giapponese e sto godendo altri spettacoli naturali, che sono i più comuni $\mathrm{e}$ i più emozionanti. Vedo che Lei ricorda ancora con nostalgia la festosa primavera nipponica.

Quanto al Sig. Luiz Trigoso, ricordo di aver visto varie notizie sui giornali, poi più nulla. Essere ora "censore" del Governo inglese a 50 scellini settimanali è un bell'esempio di come cambia il mondo. Non ha comunque molto da lamentarsi: se io avessi 50 scellini settimanali, cioè circa 100 yen al mese, sarei un lord. E' vero che i costi della vita a Tokushima non si possono paragonare a quelli di Londra: qui mangio riso, in casa vesto zimarre giapponesi e nelle mie passeggiate (generalmente notturne) uso stivali rotti e giacchette che sono in servizio da ormai cinque o sei anni... Che delizia! [15|16].

Dei mutamenti che la nostra terra sta attraversando ${ }^{16}$ ho una vaga idea dalle notizie di alcuni giornali che mi arrivano e che leggo in mancanza di meglio; e anche dalle informazioni di alcuni amici, e le Sue sono preziose. Sì, signore: la nostra terra segue il suo fato rigoroso. Leote do Rego ${ }^{17}$ è giunto ora al culmine della sua fama: io l'ho visto solo un volta, quando aveva ancora i calzoni corti; ho viaggiato una volta con suo padre, e un'altra con sua madre. Come cambia il mondo! Ho sorriso amaramente tra me e me quando poco fa ho letto che Leote do Rego comunicò al nuovo comandante della Marina militare che le guarnigioni si trovavano in stato di perfetta disciplina. Ma vada a nascondersi: perfetta

${ }^{16}$ In quei mesi il Portogallo aveva appena conosciuto le turbolente vicende governative legate a Joaquim Pimenta de Castro, cfr. nota 4.

${ }^{17}$ Jaime Daniel Leote do Rego (1867-1923), alto ufficiale di marina e quasi coetaneo di Wenceslau de Moraes, era governatore di São Tomé quando aderì alla Repubblica, nel cui parlamento fu deputato. Si oppose alla rivolta di Pimenta de Castro del 14 maggio 1915 e a quello scontro si riferisce la lettera di Wenceslau de Moraes, scritta circa due mesi dopo la rivolta. 
disciplina, dopo aver assassinato in modo infame il Secondo Comandante ed aver bombardato la città! Se fossi stato lì, già da tempo mi sarei buscato una pallottola. Ecco un altro vantaggio di Tokushima: le cannonate delle nostre navi da guerra non raggiungono la mia isola!

Sulla guerra trovo molto interessante la Sua analisi degli alleati. Ma che gente formidabile sono questi tedeschi! Se vincessero, Lei si chiede "quale sarà la futura Europa". Certo una cosa ben diversa dall'Europa attuale, ma diversa in meglio, nel senso generale dei progressi umani, perché gli Alleati - confrontati con il fuoco sacro dei teutoni - rappresentano Stati caduchi. L'azione dell'Italia, entrata tardi in guerra ingannando i suoi alleati e [16|17] quando ritiene che l'Austria sia esausta, mi dà l'idea d'una profonda canagliata. Restando sul tema della guerra, mi rallegrerei se anche questa volta la prudente Inghilterra non prestasse attenzione a quanto le dice l'ossequiente portoghese ${ }^{18}$, facendo a meno del gagliardo aiuto che insistiamo nell'offrirle. È chiaro che la maggior parte del nostro entusiasmo rivela l'ansia di percentuali e di partecipazione ai rifornimenti: il burro rancido inviato alle nostre truppe in Africa la dice lunga su questo atteggiamento.

Mi interessa molto quanto mi dice di Pedro Diniz, cui ebbe la bontà di mostrare la mia lettera, e m'impressiona molto il terribile colpo che ha appena ricevuto nell'ambito dei suoi affetti. In quel vecchio, che conosco poco, mi par di sentire un'anima eccezionale, cosa rara...

Vuole che Le parli di me? Sto vivendo qui, dove non arrivano le cannonate di Leote do Rego, il che è un gran vantaggio. Intanto le tristi notizie che costantemente mi giungono dalla mia Patria lontana, e che conto di non tornare più a vedere, mi frastornano molto. Per altro verso, soffro molto per quanto riguarda la mia povera vita sentimentale, trafitto

${ }^{18}$ Per indicare la condizione di asservimento del Portogallo all'Inghilterra, l'espressione "marreco portuguez" (p. 17) va intesa come "gobbo", cioè persona dalla schiena piegata in atteggiamento servile (e non come "anitra" o, più esattamente, "fischione"). 
dai rimpianti e per di più sempre contrariato da mille piccoli crucci della vita quotidiana. Non posso lamentarmi di nessuna persona o cosa: è il mio equilibrio psichico che è crollato, lasciandomi in balìa d'un temperamento profondamente infermo che non ha mai saputo trovare la vera rotta da seguire. Per [17|18] valutare il mio stato d'animo attraverso un esempio banale Le racconto quanto segue, giusto per farLa ridere, visto che non ha la lacrima facile. Un giorno volevo dividere 350 per 31 con due decimali, e ci riuscii solo dopo vari tentativi, perché avevo dimenticato la regola della divisione! Ma questa situazione deve durare poco, se ancora c'è coerenza nelle leggi naturali. Per me la morte sarà un gran sollievo, però sono così inetto che non me la cerco con i mezzi di cui dispongo, e che naturalmente sono molti.

E Lei che fa, Amico mio? Si stava preparando per andare in campagna, e faceva molto bene, a mio avviso. Andate in giro a piacere per il nostro Portogallo. Quello che Afonso $\operatorname{Costa}^{19}$ non riuscirà mai a distruggere è l'incanto del nostro paesaggio, la dolcezza del nostro clima, la poesia delle nostre campagne fiorite. Goda di tutto ciò, ritempri le forze e lo spirito, faccia il bucato di tutte le cattive impressioni che La tormentano. Se all'ombra di un albero si ricorderà un giorno di scrivermi una lettera, tanto meglio.

Termino qui. Resti in buona salute, si goda la sua villa e la dolce compagnia della Sua famiglia e dei Suoi amici.

Labbraccia con nostalgia il Suo Wenceslau.

Dopo la fine della Grande Guerra una lunga lettera del 1919 affronta, tra l'altro, il tema di quell'incerto dopoguerra segnato dal Trattato di Versailles, foriero d'una futura cata-

19 Afonso Augusto da Costa (1871-1937), personaggio di primo piano nella Prima Repubblica, venne eletto per la terza volta Presidente del Consiglio nel 1917. Fu arrestato per breve tempo durante il colpo di Stato di Sidonio Pais (cfr. nota 5). Il giudizio negativo di Moraes è forse dovuto allo stato d'assedio da lui dichiarato durante la "Revolução da Batata” (cfr. \$2). 
strofe bellica e, come scrive il preveggente Moraes nella lettera che segue, dell'avvento di un "despota" imposto "dal delirio delle grandi masse".

Tokushima, 9 ottobre $1919^{20}$.

Mio buon Amico, Signor Azevedo,

Ho ricevuto giorni fa la sua bella lettera del 17 di luglio. Sono stato leggermente indisposto, con allergie al volto e altri piccoli malanni, a causa del brusco cambiamento dal caldo al freddo (benché io stia meglio con il fresco). Per questo rispondo un po' in ritardo.

Vedo con dolore che il lutto che avevo notato nella sua lettera precedente rappresentava, contrariamente a quanto ritenevo, la perdita di un parente molto prossimo e certamente caro. Partecipo al suo dolore, Amico mio.

Ma torniamo alla sua bella lettera. Dico 'bella' senza esagerazione. Lei, Amico mio, non vuole essere un letterato, e ha le Sue buone ragioni. Il fatto è, però, che le Sue lettere sono belle. Ho letto molte belle lettere stampate nei libri (per esempio, The Life and Letters of Lafcadio Hearn, di Elisabeth Bisland, New York), ma tra quelle che ricevo soltanto le Sue meritano questo aggettivo.

Condivido pienamente le Sue considerazioni su quanto sta capitando nel mondo. [33|34] Mi pare che tutto vada di male in peggio. E mi pare che tutto andrà ancora peggio. Continuerà il delirio delle grandi masse, provocando disorientamenti estremi, fino a quando tutto crolli e le grandi masse si assoggettino a qualche despota che dovrà comparire. È sempre la stessa cosa: la storia si ripete. Tempo fa l'effervescenza era percepibile, ma la guerra, o meglio la Pace, ha fatto precipitare gli eventi. La storia dell'umanità si divide

${ }^{20}$ La lettera qui tradotta è in Moraes, Cartas a Polycarpo de Azevedo (cfr. nota 14), pp. 33-37. 
in periodi: sembra che siamo giunti alla fine di un periodo; ne seguirà un altro, poco diverso dagli anteriori; ma ci vorrà qualche tempo per realizzare questa transizione.

Ignoravo del tutto quanto Lei, amico mio, mi racconta di sfuggita sulle feste erotiche a Parigi per celebrare la vittoria. Che divertente! Ma feste, feste e feste, quando il futuro si presenta così nero! C'è l'Inghilterra, alle prese con l'Irlanda... e il resto. E il Belgio che si accalora a provocare l'Olanda. E l'Italia, con il suo poeta alla testa, a complicare la situazione. Vedremo o, anzi, non vedremo: per lo meno io non sarò più vivo per assistere alle prime scene del nuovo dramma che verrà rappresentato.

E il Portogallo? Il suo destino dipenderà in parte dal far parte dell'entourage, cioè di quel che starà capitando intorno a lui, e in parte dal giudizio (o dalla mancanza di giudizio) dei suoi dirigenti, dei suoi intellettuali, e anche del suo popolo. Il quadro non si annuncia allegro, senza voler essere pessimisti. [34|35]

Quanto alla Lega delle Nazioni, alla protezione dei paesi piccoli, alla libertà, alla giustizia ecc.: quanto ne abbiamo parlato!

Vuole che Le dica di me e del Giappone? Il Giappone è un caso abbastanza complicato. Io non so quello che càpita nella casa del mio vicino, figurarsi nel Giappone! Sta vivendo, le industrie vanno sviluppandosi, tutti vogliono essere ricchi ecc. La vita è carissima e tende a divenirlo ancora di più, come conseguenza... della pace. Gli scioperi si sono diffusi ovunque. Ancora pochi giorni or sono erano in sciopero 15.000 operai delle officine di costruzioni navali a Kawasaki, vicino a Kobe (credo che Lei, Amico mio, abbia visitato con me quello stabilimento). Fortunatamente l'armonia è stata ritrovata... grazie all'aumento dei salari.

Quanto a me, che posso dirLe? Sto vivendo o, piuttosto, sto morendo. Gli anni, il carattere, i dispiaceri, il triste stato d'animo che mi è proprio stanno facendo il loro lavoro. 
Quello che mi impaurisce è l'essere ancora in vita. Ma nulla di quanto Le sto dicendo deve spaventarLa, evidentemente. Trascorro i miei lunghi giorni triste e snervato, ma non per il fatto di essere a Tokushima, nella situazione che Lei più o meno immagina. Quello che mi rende triste è non avere accanto a me una persona amica. Noti che, se io continuassi nella vita attiva della marina militare, oggi sarei vice-ammiraglio, con "alcuni vantaggi materiali, ma moralmente non starei meglio": sono parole sue, e molto vere. Qui sto molto bene e non potevo trovarmi meglio in un altro luogo o in un'altra situazione esistenziale più agevole. Il male è un altro, ed è senza rimedio. [35|36] Tokushima, lontana dalle bombe e dagli intrighi patrii; la mia posizione sociale pari a zero: tutto ciò è molto buono.

Un novità. Le dicevo tempo fa che volevo desistere dallo scribacchiare per il pubblico e Lei, Amico mio, non approvò questa mia sana decisione. Quindi $\mathrm{La}$ informo che sono tornato a impugnare la penna e a scarabocchiare articoli, comunque non con grande assiduità. Ho così trovato una distrazione per il mio spirito, che è la cosa principale; se poi quegli articoli non hanno valore per gli altri, non importa, vero? Spedisco i miei prodotti inattuali a un giornaletto di provincia del Nord del Portogallo ${ }^{21}$. Ne sono già stati pubblicati due, e altri seguiranno.

Rileggendo di nuovo la Sua lettera (e la si rilegge per piacere) mi avvedo che non ho ancora risposto ad alcuni punti. Anzitutto noto che ultimamente Lei ha sofferto d'una serie di varie indisposizioni, cosa che lamento di cuore. Si liberi soprattutto della nevrastenia, che conosco per esperienza personale e che è orribile. La Sua naturale robustezza e il Suo buon senso sapranno vincere tutto, e la Sua salute tornerà, ne sono certo.

\footnotetext{
${ }^{21}$ Come altre corrispondenze, anche queste vennero probabilmente inviate a $\mathrm{O}$ Comércio do Porto.
} 
$\mathrm{Da}$ alcune sue righe ricavo che Lei sia stato nominato viceammiraglio, cosa che ignoravo. Se è così, felicitazioni! Nonostante tutto, per chi ama la propria professione, il prestigio dei galloni ha una grande forza: io stesso lo so molto bene. Qual è dunque la Sua situazione? Ha già preso servizio?

Non mi dice di essere andato, o di avere avuto intenzione di andare durante i giorni trascorsi, a passare le acque o a fare qualche escursione nel nostro Portogallo. Se [36|37] non l'ha fatto, ha fatto male. Appena può fugga da quell'immondezzaio materiale e morale che è Lisbona. Respiri dell'aria buona per un paio di settimane e vedrà che poi si sentirà meglio.

Non Le chiedo di scrivermi e di darmi molte notizie, perché non ho il diritto di infastidirLa. Ma, se giungessero qui, sarebbero ricevute come la cosa preziosa che effettivamente sono.

Addio. Mi creda sempre Suo molto devoto W. de Moraes.

La vita di Wenceslau de Moraes continuerà così come la descrive in queste e in altre sue lettere: divisa fra nostalgia del passato e attesa della morte spesso evocata, ed avvenuta il $1 .^{\circ}$ luglio 1929, dieci anni dopo la seconda lettera qui tradotta.

L'ultima fotografia di Wenceslau de Moraes venne scattata nel maggio 1929, pochi mesi prima della sua morte ${ }^{22}$. Un gruppo di tre donne con un bebé colma i due terzi dell'immagine, mentre l'oggetto della loro curiosità - il "barbaro del Sud" con cui vogliono essere fotografate - è relegato in un angolo della fotografia: esotismo al contrario. Questa immagine è quasi un simbolo della vita giapponese di Wenceslau de Moraes: egli è oggetto della curiosità, ma non della comprensione dei giapponesi intorno a lui. Come sempre ha fatto nella vita, si è isolato: nella foto, appare nell'angolo sinistro in basso, con un berretto scuro, la barba irsuta e lo sguardo fisso e intento di chi ormai vede cose che noi non vediamo.

\footnotetext{
${ }^{22}$ La fotografia di Moraes è a lato della p. 126 in Cartas intimas, cit. a nota 8.
} 